\title{
Multiple positive solutions for a fourth-order integral boundary value problem on time scales
}

\author{
Yongkun Li ${ }^{*}$ and Yanshou Dong
}

* Correspondence: yklie@ynu.edu. $\mathrm{cn}$

Department of Mathematics, Yunnan University Kunming, Yunnan 650091 People's Republic of China

\section{Abstract}

In this article, we investigate the multiplicity of positive solutions for a fourth-order system of integral boundary value problem on time scales. The existence of multiple positive solutions for the system is obtained by using the fixed point theorem of cone expansion and compression type due to Krasnosel'skill. To demonstrate the applications of our results, an example is also given in the article.

Keywords: positive solutions, fixed points, integral boundary conditions, time scales

\section{Introduction}

Boundary value problem (BVP) for ordinary differential equations arise in different areas of applied mathematics and physics and so on, the existence and multiplicity of positive solutions for such problems have become an important area of investigation in recent years, lots of significant results have been established by using upper and lower solution arguments, fixed point indexes, fixed point theorems and so on (see [1-8] and the references therein). Especially, the existence of positive solutions of nonlinear BVP with integral boundary conditions has been extensively studied by many authors (see [9-18] and the references therein).

However, the corresponding results for BVP with integral boundary conditions on time scales are still very few [19-21]. In this article, we discuss the multiple positive solutions for the following fourth-order system of integral BVP with a parameter on time scales

$$
\left\{\begin{array}{l}
x^{(4 \Delta)}(t)+\lambda f\left(t, x(t), x^{\Delta}(t), x^{\Delta \Delta}(t), y(t), y^{\Delta}(t), y^{\Delta \Delta}(t)\right)=0, \quad t \in(0, \sigma(T))_{\mathbb{T}} \\
y^{(4 \Delta)}(t)+\mu g\left(t, x(t), x^{\Delta}(t), x^{\Delta \Delta}(t), y(t), y^{\Delta}(t), y^{\Delta \Delta}(t)\right)=0, \quad t \in(0, \sigma(T))_{\mathbb{T}}, \\
x(0)=x^{\Delta}(0)=0, \\
y(0)=y^{\Delta}(0)=0, \\
a_{1} x^{\Delta \Delta}(0)-b_{1} x^{\Delta \Delta \Delta}(0)=\int_{0}^{\sigma(T)} x^{\Delta \Delta}(s) A_{1}(s) \Delta s, \\
c_{1} x^{\Delta \Delta}(\sigma(T))+d_{1} x^{\Delta \Delta \Delta}(\sigma(T))=\int_{0}^{\sigma(T)} x^{\Delta \Delta}(s) B_{1}(s) \Delta s, \\
a_{2} y^{\Delta \Delta}(0)-b_{2} y^{\Delta \Delta \Delta}(0)=\int_{0}^{\sigma(T)} y^{\Delta \Delta}(s) A_{2}(s) \Delta s, \\
c_{2} y^{\Delta \Delta}(\sigma(T))+d_{2} y^{\Delta \Delta \Delta}(\sigma(T))=\int_{0}^{\sigma(T)} y^{\Delta \Delta}(s) B_{2}(s) \Delta s,
\end{array}\right.
$$

\section{Springer}

( 2011 Li and Dong; licensee Springer. This is an Open Access article distributed under the terms of the Creative Commons Attribution License (http://creativecommons.org/licenses/by/2.0), which permits unrestricted use, distribution, and reproduction in any medium, provided the original work is properly cited. 
where $a_{i}, b_{i}, c_{i}, d_{i} \geq 0$, and $\rho_{i}=a_{i} c_{i} \sigma(T)+a_{i} d_{i}+b_{i} c_{i}>0(i=1,2), 0<\lambda, \mu<+\infty, f$, $g \in C\left((0, \sigma(T))_{\mathbb{T}} \times\left(\mathbb{R}^{+}\right)^{6}, \mathbb{R}^{+}\right), \mathbb{R}^{+}=[0,+\infty), A_{i}$ and $B_{i}$ are nonnegative and $r d$-continuous on $[0, \sigma(T)]_{\mathbb{T}}(i=1,2)$.

The main purpose of this article is to establish some sufficient conditions for the existence of at least two positive solutions for system (1.1) by using the fixed point theorem of cone expansion and compression type. This article is organized as follows. In Section 2, some useful lemmas are established. In Section 3, by using the fixed point theorem of cone expansion and compression type, we establish sufficient conditions for the existence of at least two positive solutions for system (1.1). An illustrative example is given in Section 4 .

\section{Preliminaries}

In this section, we will provide several foundational definitions and results from the calculus on time scales and give some lemmas which are used in the proof of our main results.

A time scale $\mathbb{T}$ is a nonempty closed subset of the real numbers $\mathbb{R}$.

Definition 2.1. [22] For $t \in \mathbb{T}$, we define the forward jump operator $\sigma: \mathbb{T} \rightarrow \mathbb{T}$ by $\sigma(t)=\inf \{\tau \in \mathbb{T}: \tau>t\}$, while the backward jump operator $\rho: \mathbb{T} \rightarrow \mathbb{T} b y$ $\rho(t)=\sup \{\tau \in \mathbb{T}: \tau<t\}$.

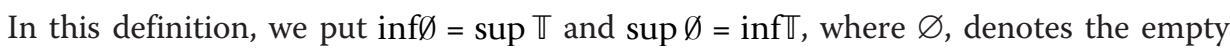
set. If $\sigma(t)>t$, we say that $t$ is right-scattered, while if $\rho(t)<t$, we say that $t$ is leftscattered. Also, if $t<\sup \mathbb{T}$ and $\sigma(t)=t$, then $t$ is called right-dense, and if $t>\inf \mathbb{T}$ and $\rho(t)=t$, then $t$ is called left-dense. We also need, below, the set $\mathbb{T}^{k}$, which is derived from the time scale $\mathbb{T}$ as follows: if $\mathbb{T}$ has a left-scattered maximum $m$, then $\mathbb{T}^{k}=\mathbb{T}-m$. Otherwise, $\mathbb{T}^{k}=\mathbb{T}$.

Definition 2.2. [22] Assume that $x: \mathbb{T} \rightarrow \mathbb{R}$ is a function and let $t \in \mathbb{T}^{k}$. Then $x$ is called differentiable at $t \in \mathbb{T}$ if there exists a $\theta \in \mathbb{R}$ such that for any given $\varepsilon>0$, there is an open neighborhood $U$ of $t$ such that

$$
\left|x(\sigma(t))-x(s)-x^{\Delta}(t)\right| \sigma(t)-s|| \leq \varepsilon|\sigma(t)-s|, \quad s \in U .
$$

In this case, $x^{\Delta}(t)$ is called the delta derivative of $x$ at $t$. The second derivative of $x(t)$ is defined by $x^{\Delta \Delta}(t)=\left(x^{\Delta}\right)^{\Delta}(t)$.

In a similar way, we can obtain the fourth-order derivative of $x(t)$ is defined by $x^{(4 \Delta)}$ $(t)=\left(\left(\left(x^{\Delta}\right)^{\Delta}\right)^{\Delta}\right)^{\Delta}(t)$.

Definition 2.3. [22] A function $f: \mathbb{T} \rightarrow$ Ris called $r d$-continuous provided it is continuous at right-dense points in Tand its left-sided limits exist at left-dense points in $\mathbb{T}$. The set of rd-continuous functions $f: \mathbb{T} \rightarrow$ Rwill be denoted by $C_{r d}(\mathbb{T})$.

Definition 2.4. [22]A function $F: \mathbb{T} \rightarrow \mathbb{R}$ is called a delta-antiderivative of $f: \mathbb{T} \rightarrow$ Rprovide $F^{\Delta}(t)=f(t)$ holds for all $t \in \mathbb{T}^{k}$. In this case we define the integral of $f$ by

$$
\int_{a}^{t} f(s)=F(t)-F(a)
$$

For convenience, we denote $I=[0, \sigma(T)]_{\mathbb{T}}, I^{\prime}=(0, \sigma(T))_{\mathbb{T}}$ and for $i=1$, 2, we set

$$
D_{1 i}=\frac{Q_{1 i}}{1-P_{1 i}}, \quad D_{2 i}=\frac{P_{2 i}}{1-Q_{2 i}}, \quad K_{1 i}=\frac{1}{1-P_{1 i}}, \quad K_{2 i}=\frac{1}{1-Q_{2 i}},
$$


where

$$
\begin{aligned}
P_{1 i} & =\int_{0}^{\sigma(T)} B_{i}(s) \frac{a_{i} s+b_{i}}{\rho_{i}} \Delta s, \quad P_{2 i}=\int_{0}^{\sigma(T)} A_{i}(s) \frac{a_{i} s+b_{i}}{\rho_{i}} \Delta s, \\
Q_{1 i} & =\int_{0}^{\sigma(T)} B_{i}(s) \frac{d_{i}+c_{i}(\sigma(T)-s)}{\rho_{i}} \Delta s, \quad Q_{2 i}=\int_{0}^{\sigma(T)} A_{i}(s) \frac{d_{i}+c_{i}(\sigma(T)-s)}{\rho_{i}} \Delta s .
\end{aligned}
$$

To establish the existence of multiple positive solutions of system (1.1), let us list the following assumptions:

$$
\left(H_{1}\right) P_{j i}, Q_{j i} \in[0,1), D_{11} D_{21} \in[0,1), D_{21} D_{22} \in[0,1), \quad j, i=1,2 .
$$

In order to overcome the difficulty due to the dependence of $f, g$ on derivatives, we first consider the following second-order nonlinear system

$$
\left\{\begin{array}{l}
u^{\Delta \Delta}(t)+\lambda f\left(t, A_{2} u, A_{1} u, A_{0} u, A_{2} v, A_{1} v, A_{0} v\right)=0, \quad t \in(0, \sigma(T))_{\mathbb{T}}, \\
v^{\Delta \Delta}(t)+\mu g\left(t, A_{2} u, A_{1} u, A_{0} u, A_{2} v, A_{1} v, A_{0} v\right)=0, \quad t \in(0, \sigma(T))_{\mathbb{T}}, \\
a_{1} u(0)-b_{1} u^{\Delta}(0)=\int_{0}^{\sigma(T)} u(s) A_{1}(s) \Delta s, \\
c_{1} u(\sigma(T))+d_{1} u^{\Delta}(\sigma(T))=\int_{0}^{\sigma(T)} u(s) B_{1}(s) \Delta s, \\
a_{2} v(0)-b_{2} v^{\Delta}(0)=\int_{0}^{\sigma(T)} v(s) A_{2}(s) \Delta s, \\
c_{2} v(\sigma(T))+d_{2} v^{\Delta}(\sigma(T))=\int_{0}^{\sigma(T)} v(s) B_{2}(s) \Delta s,
\end{array}\right.
$$

where $A_{0}$ is the identity operator, and

$$
A_{i} u(t)=\int_{0}^{t}(t-\sigma(s))^{i-1} u(s) \Delta s, \quad A_{i} v(t)=\int_{0}^{t}(t-\sigma(s))^{i-1} v(s) \Delta s, \quad i=1,2 .
$$

For the proof of our main results, we will make use of the following lemmas.

Lemma 2.1. The fourth-order system (1.1) has a solution $(x, y)$ if and only if the nonlinear system (2.1) has a solution $(u, v)$.

Proof. If $(x, y)$ is a solution of the fourth-order system (1.1), let $u(t)=x^{\Delta \Delta}(t), v(t)=$ $y^{\Delta \Delta}(t)$, then it follows from the boundary conditions of system (1.1) that

$$
A_{1} u(t)=x^{\Delta}(t), A_{2} u(t)=x(t), A_{1} v(t)=y^{\Delta}(t), A_{2} v(t)=\gamma(t) .
$$

Thus $(u, v)=\left(x^{\Delta \Delta}(t), y^{\Delta \Delta}(t)\right)$ is a solution of the nonlinear system (2.1).

Conversely, if $(u, v)$ is a solution of the nonlinear system (2.1), let $x(t)=A_{2} u(t), y(t)=$ $A_{2} v(t)$, then we have

$$
x^{\Delta}(t)=A_{1} u(t), x^{\Delta \Delta}(t)=u(t), \gamma^{\Delta}(t)=A_{1} v(t), \gamma^{\Delta \Delta}(t)=v(t),
$$


which imply that

$$
x(0)=0, x^{\Delta}(0)=0, y(0)=0, y^{\Delta}(0)=0 .
$$

Consequently, $(x, y)=\left(A_{2} u(t), A_{2} v(t)\right)$ is a solution of the fourth-order system (1.1). This completes the proof.

Lemma 2.2. Assume that $D_{11} D_{21} \neq 1$ holds. Then for any $h_{1} \in C\left(P, \mathbb{R}^{+}\right)$, the following $B V P$

$$
\left\{\begin{array}{l}
u^{\Delta \Delta}(t)+h_{1}(t)=0, \quad t \in(0, \sigma(T))_{\mathbb{T}} \\
a_{1} u(0)-b_{1} u^{\Delta}(0)=\int_{0}^{\sigma(T)} u(s) A_{1}(s) \Delta s \\
c_{1} u(\sigma(T))+d_{1} u^{\Delta}(\sigma(T))=\int_{0}^{\sigma(T)} u(s) B_{1}(s) \Delta s
\end{array}\right.
$$

has a solution

$$
u(t)=\int_{0}^{\sigma(T)} H_{1}(t, s) h_{1}(s) \Delta s,
$$

where

$$
\begin{aligned}
& H_{1}(t, s)=G_{1}(t, s)+r_{1}(t) \int_{0}^{\sigma(T)} B_{1}(\tau) G_{1}(\tau, s) \Delta \tau+r_{2}(t) \int_{0}^{\sigma(T)} A_{1}(\tau) G_{1}(\tau, s) \Delta \tau, \\
& G_{1}(t, s)=\frac{1}{\rho_{1}} \begin{cases}\left(a_{1} \sigma(s)+b_{1}\right)\left[d_{1}+c_{1}(\sigma(T)-t)\right], & \sigma(s)<t, \\
\left(a_{1} t+b_{1}\right)\left[d_{1}+c_{1}(\sigma(T)-\sigma(s))\right], & t \leq \sigma(s),\end{cases} \\
& r_{11}(t)=\frac{K_{11}\left(a_{1} t+b_{1}\right)+K_{11} D_{21}\left[d_{1}+c_{1}(\sigma(T)-t)\right]}{\rho_{1}\left(1-D_{11} D_{21}\right)}, \\
& r_{21}(t)=\frac{K_{21} D_{11}\left(a_{1} t+b_{1}\right)+K_{21}\left[d_{1}+c_{1}(\sigma(T)-t)\right]}{\rho_{1}\left(1-D_{11} D_{21}\right)} .
\end{aligned}
$$

Proof. First suppose that $u$ is a solution of system (2.3). It is easy to see by integration of $\mathrm{BVP}(2.3)$ that

$$
u^{\Delta}(t)=u^{\Delta}(0)-\int_{0}^{t} h_{1}(s) \Delta s .
$$

Integrating again, we can obtain

$$
u(t)=u(0)+t u^{\Delta}(0)-\int_{0}^{t}(t-\sigma(s)) h_{1}(s) \Delta s .
$$

Let $t=\sigma(T)$ in (2.4) and (2.5), we obtain

$$
\begin{aligned}
& u^{\Delta}(\sigma(T))=u^{\Delta}(0)-\int_{0}^{\sigma(T)} h_{1}(s) \Delta s \\
& u(\sigma(T))=u(0)+\sigma(T) u^{\Delta}(0)-\int_{0}^{\sigma(T)}(\sigma(T)-\sigma(s)) h_{1}(s) \Delta s .
\end{aligned}
$$


Substituting (2.6) and (2.7) into the second boundary value condition of system (2.3), we obtain

$$
\begin{aligned}
c_{1} u(0)+\left(c_{1} \sigma(T)+d_{1}\right) u^{\Delta}(0)= & \int_{0}^{\sigma(T)}\left[d_{1}+c_{1}(\sigma(T)-\sigma(s))\right] h_{1}(s) \Delta s \\
& +\int_{0}^{\sigma(T)} u(s) B_{1}(s) \Delta s .
\end{aligned}
$$

From (2.8) and the first boundary value condition of system (2.3), we have

$$
\begin{aligned}
u^{\Delta}(0) & =\frac{a_{1}}{\rho_{1}}\left(\int_{0}^{\sigma(T)}\left[d_{1}+c_{1}(\sigma(T)-\sigma(s))\right] h_{1}(s) \Delta s+\int_{0}^{\sigma(T)} u(s) B_{1}(s) \Delta s\right. \\
& \left.-\frac{c_{1}}{a_{1}} \int_{0}^{\sigma(T)} u(s) A_{1}(s) \Delta s\right), \\
u(0) & =\frac{b_{1}}{\rho_{1}}\left(\int_{0}^{\sigma(T)}\left[d_{1}+c_{1}(\sigma(T)-\sigma(s))\right] h_{1}(s) \Delta s+\int_{0}^{\sigma(T)} u(s) B_{1}(s) \Delta s\right. \\
& \left.-\frac{c_{1}}{a_{1}} \int_{0}^{\sigma(T)} u(s) A_{1}(s) \Delta s\right)+\frac{1}{a_{1}} \int_{0}^{\sigma(T)} u(s) A_{1}(s) \Delta s .
\end{aligned}
$$

Substituting (2.9) and (2.10) into (2.5), we have

$$
\begin{aligned}
u(t)= & \int_{0}^{\sigma(T)} G_{1}(t, s) h_{1}(s) \Delta s+\frac{a_{1} t+b_{1}}{\rho_{1}} \int_{0}^{\sigma(T)} u(s) B_{1}(s) \Delta s \\
& +\frac{d_{1}+c_{1}(\sigma(T)-t)}{\rho_{1}} \int_{0}^{\sigma(T)} u(s) A_{1}(s) \Delta s .
\end{aligned}
$$

By (2.11), we get

$$
\begin{aligned}
\int_{0}^{\sigma(T)} u(s) B_{1}(s) \Delta s= & \frac{1}{1-P_{11}} \int_{0}^{\sigma(T)} B_{1}(s) \int_{0}^{\sigma(T)} G_{1}(s, \tau) h_{1}(\tau) \Delta \tau \Delta s \\
& +\frac{Q_{11}}{1-P_{11}} \int_{0}^{\sigma(T)} u(s) A_{1}(s) \Delta s, \\
\int_{0}^{\sigma(T)} u(s) A_{1}(s) \Delta s= & \frac{1}{1-Q_{21}} \int_{0}^{\sigma(T)} B_{1}(s) \int_{0}^{\sigma(T)} G_{1}(s, \tau) h_{1}(\tau) \Delta \tau \Delta s \\
& +\frac{P_{21}}{1-Q_{21}} \int_{0}^{\sigma(T)} u(s) B_{1}(s) \Delta s .
\end{aligned}
$$


By (2.12) and (2.13), we get

$$
\begin{aligned}
\int_{0}^{\sigma(T)} u(s) A_{1}(s) \Delta s= & \frac{K_{11} D_{21}}{1-D_{11} D_{21}} \int_{0}^{\sigma(T)} B_{1}(s) \int_{0}^{\sigma(T)} G_{1}(s, \tau) h_{1}(\tau) \Delta \tau \Delta s \\
& +\frac{K_{21}}{1-D_{11} D_{21}} \int_{0}^{\sigma(T)} A_{1}(s) \int_{0}^{\sigma(T)} G_{1}(s, \tau) h_{1}(\tau) \Delta \tau \Delta s, \\
\int_{0}^{\sigma(T)} u(s) B_{1}(s) \Delta s= & \frac{K_{11}}{1-D_{11} D_{21}} \int_{0}^{T} B_{1}(s) \int_{0}^{T} G_{1}(s, \tau) h_{1}(\tau) \Delta \tau \Delta s \\
& +\frac{K_{21} D_{11}}{1-D_{11} D_{21}} \int_{0}^{\sigma(T)} A_{1}(s) \int_{0}^{\sigma(T)} G_{1}(s, \tau) h_{1}(\tau) \Delta \tau \Delta s .
\end{aligned}
$$

Substituting (2.14) and (2.15) into (2.11), we have

$$
\begin{aligned}
u(t)= & \int_{0}^{\sigma(T)} G_{1}(t, s) h_{1}(s) \Delta s \\
& +\frac{K_{11}\left(a_{1} t+b_{1}\right)+K_{11} D_{21}\left[d_{1}+c_{1}(\sigma(T)-t)\right]}{\rho_{1}\left(1-D_{11} D_{21}\right)} \int_{0}^{\sigma(T)} B_{1}(s) \int_{0}^{\sigma(T)} G_{1}(s, \tau) h_{1}(\tau) \Delta \tau \Delta s \\
& +\frac{K_{21} D_{11}\left(a_{1} t+b_{1}\right)+K_{21}\left[d_{1}+c_{1}(\sigma(T)-t)\right]}{\rho_{1}\left(1-D_{11} D_{21}\right)} \int_{0}^{\sigma(T)} A_{1}(s) \int_{0}^{\sigma(T)} G_{1}(s, \tau) h_{1}(\tau) \Delta \tau \Delta s \\
= & \int_{0}^{\sigma(T)} G_{1}(t, s) h_{1}(s) \Delta s+r_{11}(t) \int_{0}^{\sigma(T)} B_{1}(s) \int_{0}^{\sigma(T)} G_{1}(s, \tau) h_{1}(\tau) \Delta \tau \Delta s \\
& +r_{21}(t) \int_{0}^{\sigma(T)} A_{1}(s) \int_{0}^{\sigma(T)} G_{1}(s, \tau) h_{1}(\tau) \Delta \tau \Delta s \\
= & \int_{0}^{\sigma(T)} H_{1}(t, s) h_{1}(s) \Delta s .
\end{aligned}
$$

Conversely, suppose $u(t)=\int_{0}^{\sigma(T)} H_{1}(t, s) h_{1}(s) \Delta s$, then

$$
\begin{aligned}
u(t)= & \int_{0}^{\sigma(T)} G_{1}(t, s) h_{1}(s) \Delta s+r_{11}(t) \int_{0}^{\sigma(T)} B_{1}(s) \int_{0}^{\sigma(T)} G_{1}(s, \tau) h_{1}(\tau) \Delta \tau \Delta s \\
& +r_{21}(t) \int_{0}^{\sigma(T)} A_{1}(s) \int_{0}^{\sigma(T)} G_{1}(s, \tau) h_{1}(\tau) \Delta \tau \Delta s .
\end{aligned}
$$


Direct differentiation of (2.17) implies

$$
\begin{aligned}
u^{\Delta}(t)= & \frac{1}{\rho_{1}}\left(a_{1} \int_{t}^{\sigma(T)}\left[d_{1}+c_{1}(\sigma(T)-\sigma(s))\right] h_{1}(s) \Delta s-c_{1} \int_{0}^{t}\left(a_{1} \sigma(s)+b_{1}\right) h_{1}(s) \Delta s\right) \\
& +\frac{a_{1} K_{11}-c_{1} K_{11} D_{21}}{\rho_{1}\left(1-D_{11} D_{21}\right)} \int_{0}^{\sigma(T)} B_{1}(s) \int_{0}^{\sigma(T)} G_{1}(s, \tau) h_{1}(\tau) \Delta \tau \Delta s \\
& +\frac{a_{1} K_{21} D_{11}-c_{1} K_{21}}{\rho_{1}\left(1-D_{11} D_{21}\right)} \int_{0}^{\sigma(T)} A_{1}(s) \int_{0}^{\sigma(T)} G_{1}(s, \tau) h_{1}(\tau) \Delta \tau \Delta s
\end{aligned}
$$

and

$$
u^{\Delta \Delta}(t)=-h_{1}(t)
$$

and it is easy to verify that

$$
\begin{aligned}
& a_{1} u(0)-b_{1} u^{\Delta}(0)=\int_{0}^{\sigma(T)} u(s) A_{1}(s) \Delta s \\
& c_{1} u(\sigma(T))+d_{1} u^{\Delta}(\sigma(T))=\int_{0}^{\sigma(T)} u(s) B_{1}(s) \Delta s .
\end{aligned}
$$

This completes the proof.

Lemma 2.3. Assume that $D_{12} D_{22} \neq 1$ holds. Then for any $h_{2} \in C\left(I, \mathbb{R}^{+}\right)$, the following $B V P$

$$
\left\{\begin{array}{l}
v^{\Delta \Delta}(t)+h_{2}(t)=0, \quad t \in(0, \sigma(T))_{\mathbb{T}} \\
a_{2} v(0)-b_{2} v^{\Delta}(0)=\int_{0}^{\sigma(T)} v(s) A_{2}(s) \Delta s \\
c_{2} v(\sigma(T))+d_{2} v^{\Delta}(\sigma(T))=\int_{0}^{\sigma(T)} v(s) B_{2}(s) \Delta s
\end{array}\right.
$$

has a solution

$$
v(t)=\int_{0}^{\sigma(T)} H_{2}(t, s) h_{2}(s) \Delta s,
$$

where

$$
\begin{aligned}
& H_{2}(t, s)=G_{2}(t, s)+r_{12}(t) \int_{0}^{\sigma(T)} B_{2}(\tau) G_{2}(\tau, s) \Delta \tau+r_{22}(t) \int_{0}^{\sigma(T)} A_{2}(\tau) G_{2}(\tau, s) \Delta \tau, \\
& G_{2}(t, s)=\frac{1}{\rho_{2}}\left\{\begin{array}{l}
\left(a_{2} \sigma(s)+b_{2}\right)\left[d_{2}+c_{2}(\sigma(T)-t)\right], \sigma(s)<t \\
\left(a_{2} t+b_{2}\right)\left[d_{2}+c_{2}(\sigma(T)-\sigma(s))\right], t \leq \sigma(s)
\end{array}\right. \\
& r_{12}(t)=\frac{K_{12}\left(a_{2} t+b_{2}\right)+K_{12} D_{22}\left[d_{2}+c_{2}(\sigma(T)-t)\right]}{\rho_{2}\left(1-D_{12} D_{22}\right)} \\
& r_{22}(t)=\frac{K_{22} D_{12}\left(a_{2} t+b_{2}\right)+K_{22}\left[d_{2}+c_{2}(\sigma(T)-t)\right]}{\rho_{2}\left(1-D_{12} D_{22}\right)} .
\end{aligned}
$$

Proof. The proof is similar to that of Lemma 2.2 and will omit it here. 
Lemma 2.4. Suppose that $\left(H_{1}\right)$ is satisfied, for all $t, s \in I$ and $i=1,2$, we have

(i) $G_{i}(t, s)>0, H_{i}(t, s)>0$,

(ii) $\operatorname{Lim}_{i} G_{i}(\sigma(s), s) \leq H_{i}(t, s) \leq M_{i} G_{i}(\sigma(s), s)$,

(iii) $m G_{i}(\sigma(s), s) \leq H_{i}(t, s) \leq M G_{i}(\sigma(s), s)$,

where

$$
\begin{aligned}
& M_{i}=1+\overline{r_{1 i}} \int_{0}^{\sigma(T)} B_{i}(\tau) \Delta \tau+\overline{r_{2 i}} \int_{0}^{\sigma(T)} A_{i}(\tau) \Delta \tau, \quad \overline{r_{j i}}=\max _{0 \leq t \leq 1} r_{i}(t), \\
& m_{i}=1+\underline{r_{1 i}}(t) \int_{0}^{\sigma(T)} B_{i}(\tau) \Delta \tau+\underline{r_{2 i}}(t) \int_{0}^{\sigma(T)} A_{i}(\tau) \Delta \tau, \quad \underline{r_{j i}}=\min _{0 \leq t \leq 1} r_{i}(t), \\
& M=\max \left\{M_{1}, M_{2}\right\}, \quad m=\min \left\{L_{1} m_{1}, L_{2} m_{2}\right\}, \quad L_{i}=\min \left\{\frac{d_{i}}{d_{i}+c_{i}}, \frac{b_{i}}{a_{i}+b_{i}}\right\}, \quad i, j=1,2 .
\end{aligned}
$$

Proof. It is easy to verify that $G_{i}(t, s)>0, H_{i}(t, s)>0$ and $G_{i}(t, s) \leq G_{i}(\sigma(s), s)$, for all $t$, $s \in I$. Since

$$
\frac{G_{i}(t, s)}{G_{i}(\sigma(s), s)}= \begin{cases}\frac{d_{i}+c_{z}(\sigma(T)-t)}{d_{i}+c_{z}(\sigma(T)-\sigma(s))}, & \sigma(s)<t, \\ \frac{a_{i} t+b_{i}}{a_{i} \sigma(s)+b_{i}}, & \sigma(s) \geq t .\end{cases}
$$

Thus $G_{i}(t, s) / G_{i}(\sigma(s), s) \geq L_{i}$ and we have

$$
G_{i}(t, s) \geq L_{i} G_{i}(\sigma(s), s) .
$$

On the one hand, from the definition of $L_{i}$ and $m_{i}$, for all $t, s \in I$, we have

$$
\begin{aligned}
H_{i}(t, s) & =G_{i}(t, s)+r_{1 i}(t) \int_{0}^{\sigma(T)} B_{i}(\tau) G_{i}(\tau, s) \Delta \tau+r_{2 i}(t) \int_{0}^{\sigma(T)} A_{i}(\tau) G_{i}(\tau, s) \Delta \tau \\
& \left.\geq L_{i} G_{i}(\sigma(s), s)\left(1+r_{1 i}(t) \int_{0}^{\sigma(T)} B_{i}(\tau) \Delta \tau+r_{2 i}(t) \int_{0}^{\sigma(T)} A_{i}(\tau) \Delta \tau\right)\right) \\
& \geq L_{i} m_{i} G_{i}(\sigma(s), s)
\end{aligned}
$$

and on the other hand, we obtain easily that from the definition of $M_{i}$, for all $t, s \in I$,

$$
\begin{aligned}
H_{i}(t, s) & \leq G_{i}(\sigma(s), s)+r_{1 i}(t) \int_{0}^{\sigma(T)} B_{i}(\tau) G_{i}(\sigma(s), s) \Delta \tau+r_{2 i}(t) \int_{0}^{\sigma(T)} A_{i}(\tau) G_{i}(\sigma(s), s) \Delta \tau \\
& \leq M_{i} G_{i}(\sigma(s), s) .
\end{aligned}
$$

Finally, it is easy to verify that $m G_{i}(\sigma(s), s) \leq H_{i}(t, s) \leq M G_{i}(\sigma(s), s)$. This completes the proof.

Lemma 2.5. [23] Let $E$ be a Banach space and $P$ be a cone in E. Assume that $\Omega_{1}$ and $\Omega_{2}$ are bounded open subsets of $E$, such that $0 \in \Omega_{1}, \overline{\Omega_{1}} \subset \Omega_{2}$, and let $T: P \cap\left(\overline{\Omega_{2}} \backslash \Omega_{1}\right) \rightarrow$ Pbe a completely continuous operator such that either

(i) $\|T u\| \leq\|u\|, \forall u \in P \cap \partial \Omega_{1}$ and $\|T u\| \geq\|u\|, \forall u \in P \cap \partial \Omega_{2}$ or

(ii) $\|T u\| \geq\|u\|, \forall u \in P \cap \partial \Omega_{1}$ and $\|T u\| \leq\|u\|, \forall u \in P \cap \partial \Omega_{2}$ 
holds. Then $T$ has a fixed point in $P \cap\left(\overline{\Omega_{2}} \backslash \Omega_{1}\right)$.

To obtain the existence of positive solutions for system (2.1), we construct a cone $P$ in the Banach space $Q=C\left(I, \mathbb{R}^{+}\right) \times C\left(I, \mathbb{R}^{+}\right)$equipped with the norm $\|(u, v)\|=\|u\|+\|v\|=\max _{t \in I}|u|+\max _{t \in I}|v|$ by

$$
P=\left\{(u, v) \in Q \mid u(t) \geq 0, v(t) \geq 0, \min _{t \in I}(u(t)+v(t)) \geq \frac{m}{M}\|(u, v)\|\right\} .
$$

It is easy to see that $P$ is a cone in $Q$.

Define two operators $T_{\lambda}, T_{\mu}: P \rightarrow C\left(I, \mathbb{R}^{+}\right)$by

$$
\begin{aligned}
& T_{\lambda}(u, v)(t)=\lambda \int_{0}^{T} H_{1}(t, s) f\left(t, A_{2} u, A_{1} u, A_{0} u, A_{2} v, A_{1} v, A_{0} v\right) \Delta s, \quad t \in I, \\
& T_{\mu}(u, v)(t)=\mu \int_{0}^{T} H_{2}(t, s) g\left(t, A_{2} u, A_{1} u, A_{0} u, A_{2} v, A_{1} v, A_{0} v\right) \Delta s, \quad t \in I .
\end{aligned}
$$

Then we can define an operator $T: P \rightarrow C\left(I, \mathbb{R}^{+}\right)$by

$$
T(u, v)=\left(T_{\lambda}(u, v), T_{\mu}(u, v)\right), \quad \forall(u, v) \in P .
$$

Lemma 2.6. Let $\left(H_{1}\right)$ hold. Then $T: P \rightarrow P$ is completely continuous.

Proof. Firstly, we prove that $T: P \rightarrow P$. In fact, for all $(u, v) \in P$ and $t \in I$, by Lemma 2.4(i) and $\left(H_{1}\right)$, it is obvious that $T_{\lambda}(u, v)(t)>0, T_{\mu}(u, v)(t)>0$. In addition, we have

$$
\begin{aligned}
T_{\lambda}(u, v)(t) & =\lambda \int_{0}^{\sigma(T)} H_{1}(t, s) f\left(t, A_{2} u, A_{1} u, A_{0} u, A_{2} v, A_{1} v, A_{0} v\right) \Delta s \\
& \leq \lambda M \int_{0}^{\sigma(T)} G_{1}(\sigma(s), s) f\left(t, A_{2} u, A_{1} u, A_{0} u, A_{2} v, A_{1} v, A_{0} v\right) \Delta s
\end{aligned}
$$

which implies $\left\|T_{\lambda}(u, v)\right\| \leq \lambda M \int_{0}^{\sigma(T)} G_{1}(\sigma(s), s) f\left(t, A_{2} u, A_{1} u, A_{0} u, A_{2} v, A_{1} v, A_{0} v\right) \Delta s$. And we have

$$
\begin{aligned}
T_{\lambda}(u, v)(t) & \geq \lambda L_{1} m_{1} \int_{0}^{\sigma(T)} G_{1}(\sigma(s), s) f\left(t, A_{2} u, A_{1} u, A_{0} u, A_{2} v, A_{1} v, A_{0} v\right) \Delta s \\
& \geq \frac{m}{M}\left\|T_{\lambda}(u, v)\right\| .
\end{aligned}
$$

In a similar way,

$$
T_{\mu}(u, v)(t) \geq \frac{m}{M}\left\|T_{\mu}(u, v)\right\| .
$$

Therefore,

$$
\begin{aligned}
\min _{t \in I}\left(T_{\lambda}(u, v)(t)+T_{\mu}(u, v)(t)\right) & \geq \frac{m}{M}\left\|T_{\lambda}(u, v)\right\|+\frac{m}{M}\left\|T_{\mu}(u, v)\right\| \\
& =\frac{m}{M}\left\|T_{\lambda}(u, v), T_{\mu}(u, v)\right\| .
\end{aligned}
$$

This shows that $T: P \rightarrow P$. 
Secondly, we prove that $T$ is continuous and compact, respectively. Let $\left\{\left(u_{k}, v_{k}\right)\right\} \in P$ be any sequence of functions with $\lim _{k \rightarrow \infty}\left(u_{k}, v_{k}\right)=(u, v) \in P$,

$$
\begin{aligned}
\left|T_{\lambda}\left(u_{k}, v_{k}\right)(t)-T_{\lambda}(u, v)(t)\right| \leq & \lambda M_{1} \sup _{t \in I} \mid f\left(t, A_{2} u_{k}, A_{1} u_{k}, A_{0} u_{k}, A_{2} v_{k}, A_{1} v_{k}, A_{0} v_{k}\right) \\
& -f\left(t, A_{2} u, A_{1} u, A_{0} u, A_{2} v, A_{1} v, A_{0} v\right) \mid \int_{0}^{\sigma(T)} G_{1}(\sigma(s), s) \Delta s,
\end{aligned}
$$

from the continuity of $f$, we know that $\left\|T_{\lambda}\left(u_{k}, v_{k}\right)-T_{\lambda}(u, v)\right\| \rightarrow 0$ as $k \rightarrow \infty$. Hence $T_{\lambda}$ is continuous.

$T_{\lambda}$ is compact provided that it maps bounded sets into relatively compact sets. Let $\bar{f}=\sup _{t \in I^{\prime}}\left|f\left(t, A_{2} u, A_{1} u, A_{0} u, A_{2} v, A_{1} v, A_{0} v\right)\right|$, and let $\Omega$ be any bounded subset of $P$, then there exists $r>0$ such that $\|(u, v)\| \leq r$ for all $(u, v) \in \Omega$. Obviously, from (2.16), we know that

$$
T_{\lambda}(u, v)(t) \leq \lambda M \bar{f} \int_{0}^{\sigma(T)} G_{1}(\sigma(s), s) \Delta s,
$$

so, $T_{\lambda} \Omega$ is bounded for all $(u, v) \in \Omega$. Moreover, let

$$
\begin{aligned}
L_{1}^{\prime}= & \frac{\lambda \bar{f}}{\rho_{1}}\left(a_{1} \int_{0}^{\sigma(T)}\left[d_{1}+c_{1}(\sigma(T)-\sigma(s))\right] \Delta s+c_{1} \int_{0}^{\sigma(T)}\left(a_{1} \sigma(s)+b_{1}\right) \Delta s\right) \\
& +\frac{\lambda \bar{f}\left|a_{1} K_{11}-c_{1} K_{11} D_{21}\right|}{\rho_{1}\left(1-D_{11} D_{21}\right)} \int_{0}^{\sigma(T)} B_{1}(s) \int_{0}^{\sigma(T)} G_{1}(s, \tau) \Delta \tau \Delta s \\
& +\frac{\lambda \bar{f}\left|a_{1} K_{21} D_{11}-c_{1} K_{21}\right|}{\rho_{1}\left(1-D_{11} D_{21}\right)} \int_{0}^{\sigma(T)} A_{1}(s) \int_{0}^{\sigma(T)} G_{1}(s, \tau) \Delta \tau \Delta s .
\end{aligned}
$$

We have

$$
\begin{aligned}
& \quad\left|T_{\lambda}(u, v)^{\Delta}(t)\right| \\
& \leq \frac{\lambda}{\rho_{1}} \mid a_{1} \int_{t}^{\sigma(T)}\left[d_{1}+c_{1}(\sigma(T)-\sigma(s))\right] f\left(s, A_{2} u, A_{1} u, A_{0} u, A_{2} v, A_{1} v, A_{0} v\right) \Delta s \\
& \quad-c_{1} \int_{0}^{t}\left(a_{1} \sigma(s)+b_{1}\right) f\left(s, A_{2} u, A_{1} u, A_{0} u, A_{2} v, A_{1} v, A_{0} v\right) \Delta s \mid \\
& +\frac{\lambda\left|a_{1} K_{11}-c_{1} K_{11} D_{21}\right|}{\rho_{1}\left(1-D_{11} D_{21}\right)} \int_{0}^{\sigma(T)} B_{1}(s) \int_{0}^{\sigma(T)} G_{1}(s, \tau) f\left(s, A_{2} u, A_{1} u, A_{0} u, A_{2} v, A_{1} v, A_{0} v\right) \Delta \tau \Delta s \\
& +\frac{\lambda\left|a_{1} K_{21} D_{11}-c_{1} K_{21}\right|}{\rho_{1}\left(1-D_{11} D_{21}\right)} \int_{0}^{\sigma(T)} A_{1}(s) \int_{0}^{\sigma(T)} G_{1}(s, \tau) f\left(s, A_{2} u, A_{1} u, A_{0} u, A_{2} v, A_{1} v, A_{0} v\right) \Delta \tau \Delta s \\
& \leq L_{1}^{\prime} .
\end{aligned}
$$

Thus, for any $(u, v) \in \Omega$ and $\forall \varepsilon>0$, let $\delta=\frac{\varepsilon}{L^{\prime} 1}$, then for $t_{1}, t_{2} \in I,\left|t_{1}-t_{2}\right|<\delta$, we have

$$
\left|T_{\lambda}(u, v)\left(t_{1}\right)-T_{\lambda}(u, v)\left(t_{2}\right)\right| \leq L_{1}^{\prime}\left|t_{1}-t_{2}\right|<\varepsilon .
$$


So, for all $(u, v) \in \Omega, T_{\lambda} \Omega$ is equicontinuous. By Ascoli-Arzela theorem, we obtain that $T_{\lambda}: P \rightarrow P$ is completely continuous. In a similar way, we can prove that $T_{\mu}: P$ $\rightarrow P$ is completely continuous. Therefore, $T: P \rightarrow P$ is completely continuous. This completes the proof.

\section{Main results}

In this section, we will give our main results on multiplicity of positive solutions of system (1.1). In the following, for convenience, we set

$$
\begin{aligned}
& f_{\beta}=\lim _{\sum_{i=1}^{6}\left|\varphi_{i}\right| \rightarrow \beta} \inf _{t \in I^{\prime}} \frac{f\left(t, \varphi_{1}, \ldots, \varphi_{6}\right)}{q_{1}(t) \sum_{i=1}^{6}\left|\varphi_{i}\right|}, \quad f^{\alpha}=\lim _{\sum_{i=1}^{6}\left|\varphi_{i}\right| \rightarrow \alpha} \sup _{t \in I^{\prime}} \frac{f\left(t, \varphi_{1}, \ldots, \varphi_{6}\right)}{q_{2}(t) \sum_{i=1}^{6}\left|\varphi_{i}\right|}, \\
& g_{\beta}=\lim _{\sum_{i=1}^{6}\left|\varphi_{i}\right| \rightarrow \beta} \inf _{t \in I^{\prime}} \frac{f\left(t, \varphi_{1}, \ldots, \varphi_{6}\right)}{q_{3}(t) \sum_{i=1}^{6}\left|\varphi_{i}\right|}, \quad g^{\alpha}=\lim _{\sum_{i=1}^{6}\left|\varphi_{i}\right| \rightarrow \alpha} \sup _{t \in I^{\prime}} \frac{f\left(t, \varphi_{1}, \ldots, \varphi_{6}\right)}{q_{4}(t) \sum_{i=1}^{6}\left|\varphi_{i}\right|},
\end{aligned}
$$

where $q_{i}(t), q_{j}(t) \in C_{r d}\left(I, \mathbb{R}^{+}\right)$satisfy

$$
\begin{aligned}
& 0<\int_{0}^{\sigma(T)} G_{1}(\sigma(s), s) q_{i}(s) \Delta s<+\infty \quad(i=1,2), \\
& 0<\int_{0}^{\sigma(T)} G_{2}(\sigma(s), s) q_{j}(s) \Delta s<+\infty \quad(j=3,4) .
\end{aligned}
$$

Theorem 3.1. Assume that $\left(H_{1}\right)$ holds. Assume further that

$\left(H_{2}\right)$ there exist a constant $R>0$, and two functions $p_{i}(t) \in C_{r d}\left(I, R_{+}\right)$satisfying $0<\int_{0}^{\sigma(T)} G_{i}(\sigma(s), s) p_{i}(s) \Delta s<+\infty(i=1,2)$ such that

$$
\begin{aligned}
& f\left(t, \varphi_{1}, \ldots, \varphi_{6}\right) \leq R p_{1}(t), \quad \forall t \in I, \quad 0<\sum_{i=1}^{6}\left|\varphi_{i}\right| \leq R, \\
& g\left(t, \varphi_{1}, \ldots, \varphi_{6}\right) \leq R p_{2}(t), \quad \forall t \in I, \quad 0<\sum_{i=1}^{6}\left|\varphi_{i}\right| \leq R,
\end{aligned}
$$

and one of the folloeing conditions is satisfied

$\left(E_{1}\right) \lambda \in\left(\frac{M_{3}}{f_{0}}, M_{4}\right), \mu \in\left(\frac{N_{3}}{g_{\infty}}, N_{4}\right)$,

$\left(E_{2}\right) \lambda \in\left(\frac{M_{3}}{f_{\infty}}, M_{4}\right), \mu \in\left(\frac{N_{3}}{g_{0}}, N_{4}\right)$,

$\left(E_{3}\right) \lambda \in\left(\frac{M_{3}}{F_{\alpha}}, M_{4}\right), \mu \in\left(0, N_{4}\right)$,

$\left(E_{4}\right) \lambda \in\left(0, M_{4}\right), \mu \in\left(\frac{N_{3}}{G_{\alpha}}, N_{4}\right)$,

where

$$
\begin{aligned}
& M_{3}=\left(\frac{m^{2}}{M} \int_{0}^{\sigma(T)} G_{1}(\sigma(s), s) q_{1}(s) \Delta s\right)^{-1}, \quad M_{4}=\left(O_{1} M N \int_{0}^{\sigma(T)} G_{1}(\sigma(s), s) p_{1}(s) \Delta s\right)^{-1}, \\
& N_{3}=\left(\frac{m^{2}}{M} \int_{0}^{\sigma(T)} G_{2}(\sigma(s), s) q_{3}(s) \Delta s\right)^{-1}, \quad N_{4}=\left(o_{2} M N \int_{0}^{\sigma(T)} G_{2}(\sigma(s), s) p_{2}(s) \Delta s\right)^{-1}, \\
& F_{\alpha}=\min \left\{f_{0}, f_{\infty}\right\}, \quad G_{\alpha}=\left\{g_{0}, g_{\infty}\right\},
\end{aligned}
$$


$O_{1}, O_{2}$ satisfy $\frac{1}{O_{1}}+\frac{1}{O_{2}} \leq 1, N=1+\sigma(T)+(\sigma(T))^{2}$. Then system (1.1) has at least two positive solutions.

Proof. We only prove the case in which $\left(H_{2}\right)$ and $\left(E_{1}\right)$ hold, the other case can be proved similarly. Firstly, from (2.2), we have

$$
\sum_{i=0}^{2} A_{i} u(t)+\sum_{i=0}^{2} A_{i} v(t) \leq\|(u, v)\|+\sigma(T)\|(u, v)\|+(\sigma(T))^{2}\|(u, v)\|=N\|(u, v)\| .
$$

Take $R_{1}=\frac{R}{N}$, and let $\Omega_{1}=\left\{(u, v) \in Q ;\|(u, v)\|<R_{1}\right\}$. For any $t \in I,(u, v) \in \partial \Omega_{1} \cap$ $P$, it follows from $\lambda<M_{4}, \mu<N_{4}$ and $\left(H_{2}\right)$ that

$$
\begin{aligned}
T_{\lambda}(u, v)(t) & =\lambda \int_{0}^{\sigma(T)} H_{1}(t, s) f\left(s, A_{2} u, A_{1} u, A_{0} u, A_{2} v, A_{1} v, A_{0} v\right) \Delta s \\
& \leq M_{4} M \int_{0}^{\sigma(T)} G_{1}(\sigma(s), s) f\left(s, A_{2} u, A_{1} u, A_{0} u, A_{2} v, A_{1} v, A_{0} v\right) \Delta s \\
& \leq M_{4} M R \int_{0}^{\sigma(T)} G_{1}(\sigma(s), s) p_{1}(s) \Delta s \\
& =N M_{4} M R_{1} \int_{0}^{\sigma(T)} G_{1}(\sigma(s), s) p_{1}(s) \Delta s \leq \frac{1}{O_{1}} R_{1}
\end{aligned}
$$

and

$$
\begin{aligned}
T_{\mu}(u, v)(t) & =\mu \int_{0}^{\sigma(T)} H_{2}(t, s) g\left(s, A_{2} u, A_{1} u, A_{0} u, A_{2} v, A_{1} v, A_{0} v\right) \Delta s \\
& \leq N_{4} M \int_{0}^{\sigma(T)} G_{2}(\sigma(s), s) g\left(s, A_{2} u, A_{1} u, A_{0} u, A_{2} v, A_{1} v, A_{0} v\right) \Delta s \\
& \leq N_{4} M R \int_{0}^{\sigma(T)} G_{2}(\sigma(s), s) p_{2}(s) \Delta s \\
& =N_{4} M N R_{1} \int_{0}^{\sigma(T)} G_{2}(\sigma(s), s) p_{2}(s) \Delta s \leq \frac{1}{O_{2}} R_{1} .
\end{aligned}
$$

Consequently, for any $(u, v) \in \partial \Omega_{1} \cap P$, we have

$$
\|T(u, v)\|=\left\|T_{\lambda}(u, v)\right\|+\left\|T_{\mu}(u, v)\right\|<\frac{1}{\mathrm{O}_{1}} R_{1}+\frac{1}{\mathrm{O}_{2}} R_{1} \leq R_{1} .
$$

Second, from $\lambda>\frac{M_{3}}{f_{0}}$, we can choose $\varepsilon_{1}>0$ such that $\lambda f_{0}>M_{3}+\varepsilon_{1}$, then there exists $0<l_{1}<N R_{1}$ such that for any $\sum_{i=1}^{6}\left|\varphi_{i}\right|<l_{1}$ and $t \in I$,

$$
f\left(t, \varphi_{1}, \ldots, \varphi_{6}\right) \geq \frac{M_{3}+\varepsilon_{1}}{\lambda} q_{1}(t) \sum_{i=1}^{6}\left|\varphi_{i}\right| .
$$


And since

$$
\sum_{i=0}^{2} A_{i} u(t)+\sum_{i=0}^{2} A_{i} v(t) \geq u(t)+v(t) \geq \frac{m}{M}\|(u, v)\| .
$$

Take

$$
R_{2}=\frac{l_{1}}{N}<R_{1}
$$

For all $(u, v) \in \Omega_{2} \cap P$, where $\Omega_{2}=\left\{(u, v) \in Q ;\|(u, v)\|<R_{2}\right\}$, we have

$$
\sum_{i=0}^{2} A_{i} u(t)+\sum_{i=0}^{2} A_{i} v(t) \geq u(t)+v(t) \geq \frac{m}{M} R_{2} .
$$

Thus, for all $(u, v) \in \Omega_{2} \cap P$, we have

$$
\begin{aligned}
T_{\lambda}(u, v)(t) & \geq \lambda m \int_{0}^{\sigma(T)} G_{1}(\sigma(s), s) f\left(s, A_{2} u, A_{1} u, A_{0} u, A_{2} v, A_{1} v, A_{0} v\right) \Delta s \\
& \geq m\left(M_{3}+\varepsilon_{1}\right)\left(\sum_{i=0}^{2}\left|A_{i} u\right|+\sum_{i=0}^{2}\left|A_{i} v\right|\right) \int_{0}^{\sigma(T)} G_{1}(\sigma(s), s) q_{1}(s) \Delta s \\
& \geq M_{3} \frac{m^{2}}{M} R_{2} \int_{0}^{\sigma(T)} G_{1}(\sigma(s), s) q_{1}(s) \Delta s=R_{2} .
\end{aligned}
$$

Consequently, for all $(u, v) \in \Omega_{2} \cap P$, we have

$$
\|T(u, v)\| \geq\left\|T_{\lambda}(u, v)\right\| \geq\|(u, v)\| .
$$

Finally, from $\mu>N_{3} / g_{\infty}$, we can choose $\varepsilon_{2}>0$ such that $\mu g_{\infty}>N_{3}+\varepsilon_{2}$. then, there exists $l_{2}>\frac{m}{M} R$ such that for any $\sum_{i=1}^{6}\left|\varphi_{i}\right|<l_{2}$ and $t \in I$,

$$
g\left(t, \varphi_{1}, \ldots, \varphi_{6}\right) \geq \frac{N_{3}+\varepsilon_{2}}{\mu} q_{3}(t) \sum_{i=1}^{6}\left|\varphi_{i}\right| .
$$

Take

$$
R_{3}=\left(\frac{m}{M}\right)^{-1} l_{2}>R_{1} .
$$

For all $(u, v) \in \Omega_{3} \cap P$, where $\Omega_{3}=\left\{(u, v) \in Q ;\|(u, v)\|<R_{3}\right\}$, from (3.3), we have

$$
\sum_{i=0}^{2} A_{i} u(t)+\sum_{i=0}^{2} A_{i} v(t) \geq \frac{m}{M} R_{3}=l_{2} .
$$

Thus, for all $(u, v) \in \Omega_{3} \cap P$, we have

$$
\begin{aligned}
T_{\mu}(u, v)(t) & \geq \mu m \int_{0}^{\sigma(T)} G_{2}(\sigma(s), s) g\left(s, A_{2} u, A_{1} u, A_{0} u, A_{2} v, A_{1} v, A_{0} v\right) \Delta s \\
& \geq m\left(N_{3}+\varepsilon_{2}\right)\left(\sum_{i=0}^{2}\left|A_{i} u\right|+\sum_{i=0}^{2}\left|A_{i} v\right|\right) \int_{0}^{\sigma(T)} G_{2}(\sigma(s), s) q_{3}(s) \Delta s \\
& \geq N_{3} \frac{m^{2}}{M} R_{3} \int_{0}^{\sigma(T)} G_{2}(\sigma(s), s) q_{3}(s) \Delta s=R_{3} .
\end{aligned}
$$


Consequently, for all $(u, v) \in \Omega_{3} \cap P$, we have

$$
\|T(u, v)\| \geq\left\|T_{\mu}(u, v)\right\| \geq\|(u, v)\| .
$$

From (3.2), (3.4), and (ii) of Lemma 2.5, it follows that system (2.1) has one positive solution $\left(u_{1}, v_{1}\right) \in P$ with $R_{2} \leq\left\|\left(u_{1}, v_{1}\right)\right\| \leq R_{1}$. Therefore, from Lemma 2.1, it follows that system (1.1) has one positive solution $\left(x_{1}, y_{1}\right)$. In the same way, from (3.2), (3.6), and $(i)$ of Lemma 2.5, it follows that system (2.1) has one positive solution $\left(u_{2}, v_{2}\right) \in P$ with $R_{1} \leq\left\|\left(u_{2}, v_{2}\right)\right\| \leq R_{3}$. Therefore, from Lemma 2.1, it follows that system (1.1) has one positive solution $\left(x_{2}, y_{2}\right)$. Above all, system (1.1) has at least two positive solutions. This completes the proof.

Theorem 3.2. Assume that $\left(H_{1}\right)$ holds. Suppose further that

$\left(H_{3}\right)$ there exist a constant $R_{0}>0$, and two functions $w_{i}(t) \in C_{r d}\left(I, R_{+}\right)(i=1,2)$ satisfying $0<\int_{0}^{\sigma(T)} G_{i}(\sigma(s), s) w_{i}(s) \Delta s<+\infty$ such that

$$
f\left(t, \varphi_{1}, \ldots, \varphi_{6}\right) \geq R_{0} w_{1}(t), \quad \forall t \in I, \quad \sum_{i=1}^{6}\left|\varphi_{i}\right|>R_{0},
$$

or

$$
g\left(t, \varphi_{1}, \ldots, \varphi_{6}\right) \geq R_{0} w_{2}(t), \quad \forall t \in I, \quad \sum_{i=1}^{6}\left|\varphi_{i}\right|>R_{0} .
$$

Then system (1.1) has at least two positive solutions for each $\lambda \in\left(M_{5}, \frac{M_{6}}{F^{0}}\right)$ and $\mu \in\left(N_{5}, \frac{N_{6}}{G^{0}}\right)$, where

$$
\begin{aligned}
& M_{5}=\left(\frac{m^{2}}{M} \int_{0}^{\sigma(T)} G_{1}(\sigma(s), s) w_{1}(s) \Delta s\right)^{-1}, \quad N_{5}=\left(\frac{m^{2}}{M} \int_{0}^{\sigma(T)} G_{2}(\sigma(s), s) w_{2}(s) \Delta s\right)^{-1}, \\
& M_{6}=\left(O_{1} M N \int_{0}^{\sigma(T)} G_{1}(\sigma(s), s) q_{2}(s) \Delta s\right)^{-1}, \quad N_{6}=\left(o_{2} M N \int_{0}^{\sigma(T)} G_{2}(\sigma(s), s) q_{4}(s) \Delta s\right)^{-1}, \\
& F^{\alpha}=\max \left\{f^{0}, f^{\infty}\right\}<\infty, \quad G^{\alpha}=\max \left\{g^{0}, g^{\infty}\right\}<\infty .
\end{aligned}
$$

Proof. We only prove the case in which (3.7) holds. The other case in which (3.8) holds can be proved similarly.

Take

$$
R_{1}^{\prime}=\left(\frac{m}{M}\right)^{-1} R_{0}
$$

and let $\Omega_{4}=\left\{(u, v) \in Q ;\|(u, v)\|<R_{1}^{\prime}\right\}$. For any $t \in I,(u, v) \in \partial \Omega_{4} \cap P$, it follows from $\lambda>M_{5}$ and $\left(H_{3}\right)$ that

$$
\begin{aligned}
T_{\lambda}(u, v)(t) & =\lambda \int_{0}^{\sigma(T)} H_{1}(t, s) f\left(s, A_{2} u, A_{1} u, A_{0} u, A_{2} v, A_{1} v, A_{0} v\right) \Delta s \\
& \geq M_{5} m \int_{0}^{\sigma(T)} G_{1}(\sigma(s), s) f\left(s, A_{2} u, A_{1} u, A_{0} u, A_{2} v, A_{1} v, A_{0} v\right) \Delta s \\
& \geq m M_{5} R_{0} \int_{0}^{\sigma(T)} G_{1}(\sigma(s), s) w_{1}(s) \Delta s \\
& =M_{5} \frac{m^{2}}{M} R^{\prime} \int_{0}^{\sigma(T)} G_{1}(\sigma(s), s) w_{1}(s) \Delta s=R_{1}^{\prime} .
\end{aligned}
$$


Consequently, for any $(u, v) \in \partial \Omega_{4} \cap P$, we have

$$
\|T(u, v)\| \geq\left\|T_{\lambda}(u, v)\right\| \geq\|(u, v)\| .
$$

From $\lambda<\frac{M_{6}}{F^{\alpha}}, \mu<\frac{N_{6}}{G^{\alpha}}$, we know that $\lambda<\frac{M_{6}}{f^{0}}, \mu<\frac{N_{6}}{g^{0}}$, we can choose $\varepsilon_{3}>0$ such that $M_{6}-\varepsilon_{3}>0, N_{6}-\varepsilon_{3}>0$ and $\lambda f^{0}<M_{6}-\varepsilon_{3}, \mu g^{0}<N_{6}-\varepsilon_{3}$. Then there exists $0<l_{3}<N R_{0}<N R_{1}^{\prime}$ such that for any $\sum_{i=1}^{6}\left|\varphi_{i}\right|<l_{3}$ and $t \in I$,

$$
\begin{aligned}
& f\left(t, \varphi_{1}, \ldots, \varphi_{6}\right) \leq \frac{M_{6}-\varepsilon_{3}}{\lambda} q_{2}(t) \sum_{i=1}^{6}\left|\varphi_{i}\right|, \\
& g\left(t, \varphi_{1}, \ldots, \varphi_{6}\right) \leq \frac{N_{6}-\varepsilon_{3}}{\mu} q_{4}(t) \sum_{i=1}^{6}\left|\varphi_{i}\right| .
\end{aligned}
$$

Take $R_{2}^{\prime}=\frac{l_{3}}{N}<R_{1}^{\prime}$ and $\Omega_{5}=\left\{(u, v) \in Q ;\|(u, v)\|<R_{2}^{\prime}\right\}$. Then, for any $(u, v) \in \Omega_{5} \cap$ $P$, from(3.1), we have

$$
\begin{aligned}
T_{\lambda}(u, v)(t) & =\lambda \int_{0}^{\sigma(T)} H_{1}(t, s) f\left(s, A_{2} u, A_{1} u, A_{0} u, A_{2} v, A_{1} v, A_{0} v\right) \Delta s \\
& \leq \lambda M \int_{0}^{\sigma(T)} G_{1}(\sigma(s), s) f\left(s, A_{2} u, A_{1} u, A_{0} u, A_{2} v, A_{1} v, A_{0} v\right) \Delta s \\
& \leq M\left(M_{6}-\varepsilon_{3}\right)\left(\sum_{i=0}^{2}\left|A_{i} u\right|+\sum_{i=0}^{2}\left|A_{i} v\right|\right) \int_{0}^{\sigma(T)} G_{1}(\sigma(s), s) q_{2}(s) \Delta s \\
& \leq M N M_{6} R_{2}^{\prime} \int_{0}^{\sigma(T)} G_{1}(\sigma(s), s) q_{2}(s) \Delta s=\frac{1}{O_{1}} R_{2}^{\prime}
\end{aligned}
$$

and

$$
\begin{aligned}
T_{\mu}(u, v)(t) & =\mu \int_{0}^{\sigma(T)} H_{2}(t, s) g\left(s, A_{2} u, A_{1} u, A_{0} u, A_{2} v, A_{1} v, A_{0} v\right) \Delta s \\
& \leq \mu M \int_{0}^{\sigma(T)} G_{2}(\sigma(s), s) g\left(s, A_{2} u, A_{1} u, A_{0} u, A_{2} v, A_{1} v, A_{0} v\right) \Delta s \\
& \leq M\left(N_{6}-\varepsilon_{3}\right)\left(\sum_{i=0}^{2}\left|A_{i} u\right|+\sum_{i=0}^{2}\left|A_{i} v\right|\right) \int_{0}^{\sigma(T)} G_{2}(\sigma(s), s) q_{4}(s) \Delta s \\
& \leq M N N_{6} R^{\prime}{ }_{2} \int_{0}^{\sigma(T)} G_{2}(\sigma(s), s) q_{4}(s) \Delta s=\frac{1}{O_{2}} R^{\prime}{ }_{2} .
\end{aligned}
$$

Consequently, for any $(u, v) \in \partial \Omega_{5} \cap P$, we have

$$
\|T(u, v)\|=\left\|T_{\lambda}(u, v)\right\|+\left\|T_{\mu}(u, v)\right\|<\frac{1}{\mathrm{O}_{1}} R_{2}^{\prime}+\frac{1}{\mathrm{O}_{2}} R_{2}^{\prime} \leq R_{2}^{\prime} .
$$


From $\lambda<\frac{M_{6}}{F^{\alpha}}, \mu<\frac{N_{6}}{G^{\alpha}}$ we know that $\lambda<\frac{M_{6}}{f^{\infty}}, \mu<\frac{N_{6}}{g^{\infty}}$, we can choose $\varepsilon_{4}>0$ such that $M_{6}-\varepsilon_{4}>0, N_{6}-\varepsilon_{4}>0$ and $\lambda f^{\infty}<M_{6}-\varepsilon_{4}, \mu g^{\infty}<N_{6}-\varepsilon_{4}$. Then there exists $l_{4}>\frac{m}{M} R_{1}^{\prime}$ such that for any $\sum_{i=1}^{6}\left|\varphi_{i}\right|>l_{4}$ and $t \in I$,

$$
\begin{aligned}
& f\left(t, \varphi_{1}, \ldots, \varphi_{6}\right) \leq \frac{M_{6}-\varepsilon_{3}}{\lambda} q_{2}(t) \sum_{i=1}^{6}\left|\varphi_{i}\right|, \\
& g\left(t, \varphi_{1}, \ldots, \varphi_{6}\right) \leq \frac{N_{6}-\varepsilon_{3}}{\mu} q_{4}(t) \sum_{i=1}^{6}\left|\varphi_{i}\right| .
\end{aligned}
$$

Take $R_{3}^{\prime}=\left(\frac{m}{M}\right)^{-1} l_{4}>R_{1}^{\prime}$ and let $\Omega_{6}=\left\{(u, v) \in Q ;\|(u, v)\|<R_{3}^{\prime}\right\}$. Then, for any $(u, v)$ $\in \Omega_{6} \cap P$, we have

$$
\begin{aligned}
T_{\lambda}(u, v)(t) & =\lambda \int_{0}^{\sigma(T)} H_{1}(t, s) f\left(s, A_{2} u, A_{1} u, 3 A_{0} u, A_{2} v, A_{1} v, A_{0} v\right) \Delta s \\
& \leq \lambda M \int_{0}^{\sigma(T)} G_{1}(\sigma(s), s) f\left(s, A_{2} u, A_{1} u, A_{0} u, A_{2} v, A_{1} v, A_{0} v\right) \Delta s \\
& \leq M\left(M_{6}-\varepsilon_{3}\right)\left(\sum_{i=0}^{2}\left|A_{i} u\right|+\sum_{i=0}^{2}\left|A_{i} v\right|\right) \int_{0}^{\sigma(T)} G_{1}(\sigma(s), s) q_{2}(s) \Delta s \\
& \leq M N M_{6} R^{\prime} \int_{0}^{\sigma(T)} G_{1}(\sigma(s), s) q_{2}(s) \Delta s=\frac{1}{O_{1}} R_{3}^{\prime}
\end{aligned}
$$

and

$$
\begin{aligned}
T_{\mu}(u, v)(t) & =\mu \int_{0}^{\sigma(T)} H_{2}(t, s) g\left(s, A_{2} u, A_{1} u, A_{0} u, A_{2} v, A_{1} v, A_{0} v\right) \Delta s \\
& \leq \mu M \int_{0}^{\sigma(T)} G_{2}(\sigma(s), s) g\left(s, A_{2} u, A_{1} u, A_{0} u, A_{2} v, A_{1} v, A_{0} v\right) \Delta s \\
& \leq M\left(N_{6}-\varepsilon_{3}\right)\left(\sum_{i=0}^{2}\left|A_{i} u\right|+\sum_{i=0}^{2}\left|A_{i} v\right|\right) \int_{0}^{\sigma(T)} G_{2}(\sigma(s), s) q_{4}(s) \Delta s \\
& \leq M N N_{6} R^{\prime}{ }_{3} \int_{0}^{\sigma(T)} G_{2}(\sigma(s), s) q_{4}(s) \Delta s=\frac{1}{O_{2}} R_{3}^{\prime} .
\end{aligned}
$$

Consequently, for any $(u, v) \in \partial \Omega_{6} \cap P$, we have

$$
\|T(u, v)\|=\left\|T_{\lambda}(u, v)\right\|+\left\|T_{\mu}(u, v)\right\|<\frac{1}{O_{1}} R_{3}^{\prime}+\frac{1}{O_{2}} R_{3}^{\prime} \leq R_{3}^{\prime} .
$$

From (3.9), (3.10) and (i) of Lemma 2.5, it follows that system (2.1) has one positive solution $\left(u_{1}, v_{1}\right) \in P$ with $R_{1}^{\prime} \leq\left\|\left(u_{1}, v_{1}\right)\right\| \leq R_{2}^{\prime}$. Therefore, from Lemma 2.1, it follows that system (1.1) has one positive solution $\left(x_{1}, y_{1}\right)$. In the same way, from (3.9), (3.11) and (ii) of Lemma 2.5, it follows that system (2.1) has one positive solution $\left(u_{2}, v_{2}\right) \in$ 
$P$ with $R_{1}^{\prime} \leq\left\|\left(u_{2}, v_{2}\right)\right\| \leq R_{3}^{\prime}$. Therefore, from Lemma 2.1, it follows that system (1.1) has one positive solution $\left(x_{2}, y_{2}\right)$. Above all, system (1.1) has at least two positive solutions. This completes the proof.

\section{An example}

Consider the following BVP with integral boundary conditions:

$$
\left\{\begin{array}{l}
x^{(4 \Delta)}(t)+\lambda f\left(t, x(t), x^{\Delta}(t), x^{\Delta \Delta}(t), y(t), y^{\Delta}(t), y^{\Delta \Delta}(t)\right)=0, \quad t \in(0, \sigma(T))_{\mathbb{T}}, \\
y^{(4 \Delta)}(t)+\mu g\left(t, x(t), x^{\Delta}(t), x^{\Delta \Delta}(t), y(t), y^{\Delta}(t), y^{\Delta \Delta}(t)\right)=0, \quad t \in(0, \sigma(T))_{\mathbb{T}}, \\
x(0)=x^{\Delta}(0)=0, \\
y(0)=y^{\Delta}(0)=0, \\
x^{\Delta \Delta}(0)-x^{\Delta \Delta \Delta}(0)=\int_{0}^{\sigma(T)} x^{\Delta \Delta}(s) A_{1}(s) \Delta s, \\
x^{\Delta \Delta}(\sigma(T))+x^{\Delta \Delta \Delta}(\sigma(T))=\int_{0}^{\sigma(T)} x^{\Delta \Delta}(s) B_{1}(s) \Delta s, \\
y^{\Delta \Delta}(0)-y^{\Delta \Delta \Delta}(0)=\int_{0}^{\sigma(T)} y^{\Delta \Delta}(s) A_{2}(s) \Delta s, \\
y^{\Delta \Delta}(\sigma(T))+y^{\Delta \Delta \Delta}(\sigma(T))=\int_{0}^{\sigma(T)}(s) B_{2}(s) \Delta s,
\end{array}\right.
$$

where $A_{1}(t)=B_{1}(t)=t, A_{2}(t)=B_{2}(t)=t / 2$ and

$$
\begin{aligned}
& f\left(t, \phi_{1}, \phi_{2}, \phi_{3}, \phi_{4}, \phi_{5}, \phi_{6}\right)=2 t\left(\sum_{i=1}^{6} \phi_{i}\right)^{\frac{1}{2}}, \quad t \in(0, \sigma(T))_{\mathbb{J}}, \quad \phi_{i} \geq 0, \quad i=1, \ldots, 6, \\
& g\left(t, \phi_{1}, \phi_{2}, \phi_{3}, \phi_{4}, \phi_{5}, \phi_{6}\right)=\frac{t}{2}\left(\sum_{i=1}^{6} \phi_{i}\right)^{3}, \quad t \in(0, \sigma(T))_{\mathbb{V}}, \quad \phi_{i} \geq 0, \quad i=1, \ldots, 6 .
\end{aligned}
$$

we choose $O_{1}=2, O_{2}=4, R=1, p_{1}(t)=2 t, p_{2}(t)=\frac{t}{2}, q_{1}(t)=q_{3}(t)=1$. It is easy to check that $f_{0}=g_{\infty}=\infty,\left(H_{1}\right),\left(H_{2}\right)$ and $\left(E_{1}\right)$ are satisfied. Therefore, by Theorem 3.1, system (4.1) has at least two positive solutions for each $\lambda \in\left(0, M_{4}\right), \mu \in\left(0, N_{4}\right)$.

\section{Acknowledgements}

This study was supported by the National Natural Sciences Foundation of People's Republic of China under Grant 10971183.

\section{Authors' contributions}

All authors contributed equally to the manuscript and typed, read and approved the final manuscript.

\section{Competing interests}

The authors declare that they have no competing interests.

Received: 9 November 2011 Accepted: 29 December 2011 Published: 29 December 2011

\section{References}

1. Yuan, C, Jiang, D, O'Regan, D: Existence and uniqueness of positive solutions for fourth-order nonlinear singular continuous and discrete boundary value problems. Appl Math Comput. 203, 194-201 (2008). doi:10.1016/j. amc.2008.04.034 
2. Yang, Z: Existence and uniqueness of positive solutions for a higher order boundary value problem. Comput Math Appl. 54, 220-228 (2007). doi:10.1016/j.camwa.2007.01.018

3. Zhang, $X$, Feng, M: Positive solutions for a class of $2 n$ th-order singular boundary value problems. Nonlinear Anal. 69 , 1287-1298 (2008). doi:10.1016/j.na.2007.06.029

4. Zhao, J, Ge, W: A necessary and sufficient condition for the existence of positive solutions to a kind of singular threepoint boundary value problem. Nonlinear Anal. 71, 3973-3980 (2009). doi:10.1016/j.na.2009.02.067

5. Lian, $\mathrm{H}$, Pang, $\mathrm{H}, \mathrm{Ge}, \mathrm{W}$ : Triple positive solutions for boundary value problems on infinite intervals. Nonlinear Anal. 67, 2199-2207 (2007). doi:10.1016/j.na.2006.09.016

6. Wei, Z, Zhang, M: Positive solutions of singular sub-linear boundary value problems for fourth-order and second-order differential equation systems. Appl Math Comput. 197, 135-148 (2008). doi:10.1016/j.amc.2007.07.038

7. Bai, Z: Positive solutions of some nonlocal fourth-order boundary value problem. Appl Math Comput. 215, 4191-4197 (2010). doi:10.1016/j.amc.2009.12.040

8. Zhu, F, Liu, L, Wu, Y: Positive solutions for systems of a nonlinear fourth-order singular semipositone boundary value problems. Appl Math Comput. 216, 448-457 (2010). doi:10.1016/j.amc.2010.01.038

9. Kang, $P, W e i, Z, X u, J$ : Positive solutions to fourth-order singular boundary value problems with integral boundary conditions in abstract spaces. Appl Math Comput. 206, 245-256 (2008). doi:10.1016/j.amc.2008.09.010

10. Feng, M: Existence of symmetric positive solutions for a boundary value problem with integral boundary conditions. Appl Math Lett. 24, 1419-1427 (2011). doi:10.1016/j.aml.2011.03.023

11. Zhang, $\mathrm{X}, \mathrm{Ge}, \mathrm{W}$ : Positive solutions for a class of boundary-value problems with integral boundary conditions. Comput Math Appl. 58, 203-215 (2009). doi:10.1016/j.camwa.2009.04.002

12. Feng, $\mathrm{M}, \mathrm{Ji}, \mathrm{D}, \mathrm{Ge}, \mathrm{W}$ : Positive solutions for a class of boundary-value problem with integral boundary conditions in Banach spaces. J Comput Appl Math. 222, 351-363 (2008). doi:10.1016/j.cam.2007.11.003

13. Zhao, J, Wang, P, Ge, W: Existence and nonexistence of positive solutions for a class of third order BVP with integral boundary conditions in Banach spaces. Commun Nonlinear Sci Numer Simulat. 16, 402-413 (2011). doi:10.1016/j. cnsns.2009.10.011

14. Yang, Z: Existence and uniqueness of positive solutions for an integral boundary value problem. Nonlinear Anal. 69, 3910-3918 (2008). doi:10.1016/..na.2007.10.026

15. Kong, L: Second order singular boundary value problems with integral boundary conditions. Nonlinear Anal. 72, 2628-2638 (2010). doi:10.1016/j.na.2009.11.010

16. Jankowski, T: Positive solutions for fourth-order differential equations with deviating arguments and integral boundary conditions. Nonlinear Anal. 73, 1289-1299 (2010). doi:10.1016/j.na.2010.04.055

17. Boucherif, A: Second-order boundary value problems with integral boundary conditions. Nonlinear Anal. 70, 364-371 (2009). doi:10.1016/j.na.2007.12.007

18. Zhang, X, Feng, M, Ge, W: Existence results for nonlinear boundary-value problems with integral boundary conditions in Banach spaces. Nonlinear Anal. 69, 3310-3321 (2008). doi:10.1016/j.na.2007.09.020

19. Anderson, DR, Tisdell, CC: Third-order nonlocal problems with sign-changing nonlinearity on time scales. Electron J Diff Equ. 2007(19), 1-12 (2007)

20. Li, YK, Shu, J: Multiple positive solutions for first-order impulsive integral boundary value problems on time scales. Bound Value Probl. 2011, 12 (2011). doi:10.1186/1687-2770-2011-12

21. $L i, Y K, S h u, J:$ Solvability of boundary value problems with Riemann-Stieltjes -integral conditions for second-order dynamic equations on time scales at resonance. Adv Diff Equ. 2011, 42 (2011). doi:10.1186/1687-1847-2011-42

22. Bohner, M, Peterson, A: Dynamic Equations on Time Scales, An introduction with Applications. Birkhauser, Boston (2001)

23. Guo, D, Lakshmikantham, V: Nonlinear Problems in Abstract Cones. Academic Press, New york (1988)

doi:10.1186/1687-2770-2011-59

Cite this article as: $\mathrm{Li}$ and Dong: Multiple positive solutions for a fourth-order integral boundary value problem on time scales. Boundary Value Problems 2011 2011:59.

\section{Submit your manuscript to a SpringerOpen ${ }^{\ominus}$ journal and benefit from:}

- Convenient online submission

- Rigorous peer review

- Immediate publication on acceptance

- Open access: articles freely available online

- High visibility within the field

- Retaining the copyright to your article

Submit your next manuscript at $\gg$ springeropen.com 